\section{Prevalência de diarreia em \\ crianças e condições de \\ saneamento e moradia em áreas \\ periurbanas de Guarulhos, SP*}

\section{Diarrhea in children and sanitation and housing conditions in periurban areas in the city of Guarulhos, SP}

\section{Mariana Gutierres Arteiro da Paz'}

Márcia Furquim de Almeida"

Wanda Maria Risso Günther'II

'Programa de Pós-Graduação em Ciência Ambiental do Instituto de Eletrotécnica e Energia da Universidade de São Paulo

"Departamento de Epidemiologia da Faculdade de Saúde Pública da Universidade de São Paulo

"' Departamento de Saúde Ambiental da Faculdade de Saúde Pública da Universidade de São Paulo

\section{Resumo}

Foi realizado estudo transversal em área periurbana do Município de Guarulhos, SP, atendida pelo Programa Saúde da Família (PSF) para verificar associação entre ocorrência de diarreia em crianças de 0-2 anos de idade e características da criança, condições de saneamento e tipo de moradia. Os dados foram coletados nas fichas de registro do PSF. Foi realizada análise de regressão logística multivariada, a qual indicou a interação Moradia*Esgoto (outros materiais e ausência de rede de esgoto, $p<0,001$ ) e idade (4 - 9 meses, $\mathrm{p}=0,054$; e 10 meses e mais, $p=0,008$ ) como fatores de risco para ocorrência de diarreia. Em localidades com falta de dados para compor indicadores de saneamento básico que permitem identificar populações que vivem situações de risco para a ocorrência de diarréia, as informações coletadas pelo PSF podem se constituir em excelente ferramenta para identificação de núcleos populacionais com precárias condições de habitação e saneamento.

Palavras-Chave: Diarreia. Saneamento básico. Programa Saúde da Família. Área periurbana. Estudo epidemiológico. Saúde ambiental.

*Pesquisa de Mestrado realizada no período de 2005 a 2007 no Departamento de Saúde Ambiental da Faculdade de Saúde Pública/USP. A dissertação intitulada "Estudo epidemiológico em localidade periurbana no Município de Guarulhos, SP: Acesso ao saneamento e condições de saúde de crianças" foi defendida em 2007. Auxílio à pesquisa de campo e bolsa de mestrado - Agência Financiadora: ICO-DEV - International Cooperation with Developing Countries mediante contrato ICA4-CT-2002-10061.

Correspondência: Mariana Gutierres Arteiro da Paz. Rua Joaquim Nabuco, 1699, São Paulo CEP 04621-005. E-mail: mariana_gutierres@yahoo.com.br 


\section{Abstract}

A cross-sectional study was carried out to identify the association between diarrhea in 0-2 year-old children and children's characteristics, access to sanitation and housing conditions in a periurban area served by the Family Health Program, in the city of Guarulhos, SP. Data were obtained from FHP registration forms. Multiple logistic regression showed interaction for Housing*Sewage (other materials and non collected wastewater, $\mathrm{p}<0.001)$, age group ( 4 - 9 months old, $p=0.054 ; 10$ months and older, $\mathrm{p}=0.008$ ) as risk factors for diarrhea. Information collected by the Family Health Program could be an excellent tool to identify populations with poor housing and sanitation conditions at locations where sanitation indicators are not efficient to identify populations living at risk.

Keywords: Diarrhea. Sanitation. Family Health Program. Periurban area. Epidemiologic study. Environmental health.

\section{Introdução}

A diarreia é um grave problema de saúde pública intimamente associado às condições de higiene e da água utilizada ${ }^{1}$. Relatório da OMS aponta a diarreia como a segunda maior responsável por óbito na infância representando em torno de 1,5 milhões de mortes anuais de crianças de até 5 anos $^{2}$. Em dados globais, as doenças diarréicas estão entre as maiores causas de morbidade e mortalidade em países em desenvolvimento ${ }^{3}$, onde são frequentes e podem ser fatais ${ }^{4}$, principalmente em crianças jovens ${ }^{5}$. Nesses países, estima-se que 1,5\% das mortes de recém-nascidos em 1993 foram causadas por doenças diarréicas $^{4}$ e, somente na América Latina e Caribe, foram responsáveis por $7,1 \%$ dos óbitos no período de 1998 a $2002^{6}$. A transmissão de doenças infecciosas como a diarreia é um processo complexo, com muitos determinantes ${ }^{7}$; ainda assim, sabe-se que $88 \%$ das mortes por diarreia são atribuídas à água não potável, saneamento inadequado e higiene precária ${ }^{2}$. Por isso, entre as medidas de prevenção da diarreia infantil estão o provimento de água, em quantidade e qualidade; afastamento e tratamento de efluentes domiciliares; e promoção do saneamento em toda comunidade ${ }^{2}$. Esses serviços devem ser promovidos pelo setor de infraestrutura urbana, com participação do setor de saúde pública (competência do Sistema Único de Saúde-SUS) e da comunidade, como previsto na Constituição Federal de $1988^{8}$.

Em muitas localidades brasileiras, populações excluídas de infraestrutura urbana, como serviços de saneamento e equipamentos públicos, são atendidas pelo Programa de Saúde da Família (PSF), criado em 1994, como forma alternativa de promoção da saúde no país. Por meio de equipes de Agentes Comunitários de Saúde (ACS), as famílias são visitadas periodicamente e informações sobre condições da moradia, de saúde e da situação familiar são colhidas e registradas para compor o Sistema de Informação da Atenção Básica (SIAB). Os ACS acompanham mensalmente 
as famílias e registram as informações sobre condições de moradia, saneamento e saúde das crianças de 0 a 2 anos de idade.

Em áreas periurbanas, os serviços de saneamento geralmente não estão disponíveis de forma equitativa para toda a população; portanto, em muitas localidades o acesso não existe ou se dá de modo irregular. O acesso precário é responsável por grande parte da ocorrência de doenças diarréicas e está associado à pobreza ${ }^{9}$.

O estudo teve por objetivo verificar se há associação entre a variável prevalência de diarreia e as variáveis características da criança (sexo, idade, estado nutricional, aleitamento materno e peso ao nascer), condições de saneamento (abastecimento de água, tratamento de água no domicílio e coleta dos esgotos sanitários) e tipo de moradia.

\section{Métodos}

\section{Área e população estudada}

Em um município da Região Metropolitana de São Paulo (RMSP), foi identificado assentamento periurbano de baixa renda com PSF implantado desde 2005. Tendo em vista que o PSF pode ser um instrumento de identificação de áreas prioritárias para investimento em saneamento, foi realizado estudo transversal na área selecionada para verificar a possibilidade de utilização das informações levantadas pelos ACS na interface ambiente-saúde. A área de estudo é representada pelo bairro Recreio de São Jorge, município de Guarulhos, SP. A localidade é atendida pela Unidade de Saúde Recreio de São Jorge, do PSF, e localizada na Área de Proteção de Manancial do Reservatório Cabuçu. Em 2006, período de interesse para a pesquisa, o Recreio de São Jorge tinha população estimada de 17.502 habitantes e índices de saneamento insatisfatórios, com $88,7 \%$ dos domicílios servidos pelo sistema de abastecimento público de água e apenas $16,9 \%$ por coleta pública de esgotos. Considerando o número dos registros no PSF para o período de maio de 2005 a abril de 2006, foram estudadas todas as crianças na faixa etária de 0 a 2 anos durante o período de referência (817 crianças), assim como as famílias cadastradas responsáveis por estas crianças (771 famílias).

\section{Variáveis utilizadas e coleta dos dados}

A variável dependente é a ocorrência de diarreia em crianças de 0 a 2 anos de idade. Este indicador é utilizado na maioria de estudos para avaliar impactos do saneamento na saúde pública ${ }^{10}$, por sua capacidade de resposta, viabilidade em seu uso e fácil determinação da enfermidade ${ }^{11}$. As variáveis de exposição referem-se às características da criança, às condições de saneamento e da moradia. Como fontes de informação foram utilizadas a Ficha A (formulário de cadastro das famílias) e a Ficha C (formulário de acompanhamento das condições de saúde e seguimento médico de crianças menores de dois anos).

Da Ficha A foram utilizadas as variáveis:

- tipo de material utilizado na construção do domicílio: madeira, material aproveitado, tijolo e outros;

- número de cômodos no domicílio;

- número de pessoas residentes no domicílio;

- adultos residentes no domicílio, por faixa etária;

- condição de abastecimento de água: por rede pública, por poço ou nascente e outros;

- tipo de tratamento de água no domicílio: por filtração, fervura, cloração ou sem tratamento;

- destino de lixo: por coleta pública; queimado ou enterrado; e a céu aberto; e

- destino dos esgotos: coleta por rede pública; fossa; e a céu aberto.

A variável destino do lixo não fez parte do estudo, pois segundo informações do SIAB, 99\% das famílias no local eram servidas por coleta pública municipal. As informações contidas na Ficha A foram coletadas pelos ACS entre março e abril de 2005, quando o programa foi implantado, sendo que não houve intervenções sanitárias 
e educacionais no período de referência (maio de 2005 a abril de 2006).

Da Ficha C, foram utilizadas as variáveis:

- idade da criança (faixa etária);

- peso ao nascer;

- estado nutricional (apresentou desnutrição no período, sim ou não); e

- aleitamento materno (exclusivo ou misto).

A variável idade foi estratificada em três faixas etárias: as faixas de 0-3 meses, 4-9 meses e 10 meses e mais. As informações sobre características das crianças foram colhidas mensalmente pelos ACS em visita domiciliar.

\section{Análise dos dados}

Inicialmente foi realizada análise univariada entre o desfecho ocorrência de diarreia e as variáveis ambientais e de saúde da criança para a pré-seleção de variáveis, utilizando-se como ponto de corte o valor de $\mathrm{p}<0,2$ (Tabela 1). A medida de associação utilizada foi a Razão de Prevalência (RP) para um Intervalo de Confiança (IC) de 95\%. Para calcular a RP da idade e a ocorrência de diarreia, foi utilizada a RP de Mantel e Haenszel para IC de $95 \%{ }^{12}$. Essa fase da análise foi utilizada para identificar as variáveis que deveriam ser utilizadas na análise de regressão logística. Na análise multivariada utilizou-se o Modelo de Regressão Logística Multivariado, no programa estatístico Intercooled STATA 9. Foram feitos três ajustes até o modelo final, selecionando as variáveis que se associaram ao desfecho com nível de significância estatística <0,05.

\section{Resultados}

Durante o período analisado foram registrados 4.048 casos de diarreia em crianças de 0 a 2 anos. Com relação às características das crianças e das famílias na análise univariada, identificou-se que a maior prevalência de diarreia encontrava-se na idade de 4 a 9 meses. Tomando-se este grupo etário como referência, verificou-se que as idades 0 a 3 meses ou 10 meses e

Tabela 1 - Características da criança e prevalência de diarreia em crianças de 0 a 2 anos de idade no Recreio de São Jorge, Guarulhos, SP, maio de 2005 a abril de 2006.

Table 1 - Child characteristics and prevalence of diarrhea in 0-2 year-old children living in Recreio de São Jorge, Guarulhos, SP, May 2005 to April 2006.

\begin{tabular}{|c|c|c|c|c|c|c|c|c|c|}
\hline \multicolumn{2}{|c|}{ Características das crianças } & \multicolumn{4}{|c|}{ Ocorrência de diarreia } & \multirow[t]{3}{*}{ Total $\mathrm{N}^{\circ}$} & \multirow[t]{3}{*}{$\mathrm{RP}$} & \multirow[t]{3}{*}{ IC (95\%) } & \multirow[t]{3}{*}{$p$} \\
\hline & & \multicolumn{2}{|c|}{ Sim } & \multicolumn{2}{|c|}{ Não } & & & & \\
\hline & & $\mathrm{N}^{\circ}$ & $\%$ & $\mathrm{~N}^{\circ}$ & $\%$ & & & & \\
\hline \multirow[t]{2}{*}{ Desnutrição } & Sim & 5 & 22,7 & 17 & 77,3 & 22 & 1,49 & 0,$68 ; 3,28$ & 0,337 \\
\hline & Não & 121 & 15,2 & 673 & 84,8 & 794 & 1 & & \\
\hline \multirow[t]{2}{*}{ Aleitamento } & Misto & 89 & 16,3 & 458 & 83,7 & 547 & 1,07 & 0,$75 ; 1,54$ & 0,696 \\
\hline & Exclusivo & 35 & 15,2 & 196 & 84,8 & 231 & 1 & & \\
\hline \multirow[t]{2}{*}{ Sexo } & Masculino & 63 & 17,4 & 299 & 82,6 & 362 & 1,14 & 0,$82 ; 1,58$ & 0,433 \\
\hline & Feminino & 59 & 15,3 & 327 & 84,7 & 386 & 1 & & \\
\hline \multirow[t]{3}{*}{ Idade (em meses) } & 0 a 3 & 5 & 6,0 & 79 & 94,0 & 84 & ${ }^{*} 0,25$ & 0,$10 ; 0,06$ & $<0,001$ \\
\hline & 4 a 9 & 51 & 23,9 & 162 & 76,1 & 213 & 1 & & \\
\hline & 10 meses e mais & 70 & 15,4 & 386 & 84,6 & 456 & $* 0,53$ & 0,$38 ; 0,73$ & $<0,001$ \\
\hline \multirow[t]{2}{*}{ Peso ao nascer } & $<2500 \mathrm{~g}$ & 15 & 24,6 & 46 & 75,4 & 61 & 1,34 & 0,$74 ; 2,79$ & 0,242 \\
\hline & $\geq 2500 \mathrm{~g}$ & 102 & 18,4 & 452 & 81,6 & 554 & 1 & & \\
\hline
\end{tabular}

Notas: * Para a variável idade foi calculada a Razão de Prevalência de Mantel e Haenszel - RPMH

Ausência de informação: Desnutrição: 1; Aleitamento: 39; Sexo: 69; Peso ao nascer: 202

Notes: * To evaluate age, Mantel and Haenzel Prevalence Rate-RPMH was calculated

Without information: Malnutrition: 1; Breastfeeding: 39; Sex: 69; Birth weight: 202 
mais apresentavam efeito protetor para a diarreia. A idade estava associada à presença de diarreia. Observou-se associação entre a ocorrência de desnutrição e diarreia, e a variável presença de idosos na família também foi associada à desnutrição; todas as crianças desnutridas (22) eram residentes em domicílios onde havia presença de adultos acima de 55 anos de idade $\left(\mathrm{chi}^{2}=784,00 ; \mathrm{p}<\right.$ 0,001 ). Devido à presença de colinearidade entre essas variáveis, a presença de idosos não foi contemplada no modelo de regressão, enquanto que a ocorrência de desnutrição permaneceu na análise. A variável peso ao nascer também não compôs o modelo, devido à elevada perda de registro (24,7\%).

Das variáveis de exposição que retratam as condições de saneamento do domicílio e da moradia, a construção de domicílios por materiais que não o tijolo pode ser considerada fator de risco para a ocorrência de diarreia nas crianças $(\mathrm{p}<0,001)$ (Tabela 2). Esta variável, que retrata as condições socioeconômicas da família, está altamente correlacionada com o destino do esgoto sanitário, quando não coletado por rede pública ( $\mathrm{p}<0,001)$. Apenas uma família cuja moradia foi construída com outros materiais é servida pela rede coletora de esgotos; as demais possuem fossas ou lançam o esgoto sanitário a céu aberto. Por essa alta correlação encontrada entre as variáveis moradia e destino do esgoto sanitário foi criada uma variável de interação: moradia*esgoto para integrar o modelo de regressão.

No primeiro ajuste das variáveis no modelo de regressão logística, três variáveis apresentaram associação estatisticamente significante com o desfecho: presença de desnutrição ( $\mathrm{p}=0,020)$, idade (10 meses e mais) ( $p=0,056)$ e moradia ${ }^{*}$ esgoto ( $\mathrm{p}<0,001)$ como fatores de risco. No segundo ajuste, que considerou apenas as variáveis estatisticamente significantes para a ocorrência de diarreia, a variável desnutrição foi excluída do modelo.

O modelo final apresentou as variáveis: moradia*esgoto (outros materiais utilizados

Tabela 2 - Acesso ao saneamento e condições de moradia e prevalência de diarreia em crianças de 0 a 2 anos de idade no Recreio de São Jorge, Guarulhos, SP, maio de 2005 a abril de 2006.

Table 2 - Access to sanitation, housing conditions and prevalence of diarrhea in 0-2 year-old children living in Recreio de São Jorge, Guarulhos, SP, May 2005 to April 2006.

\begin{tabular}{|c|c|c|c|c|c|c|c|c|c|}
\hline \multirow[t]{3}{*}{ Características do domicílio } & & \multicolumn{4}{|c|}{ Ocorrência de diarreia } & \multirow[t]{3}{*}{ Total $\mathrm{N}^{\circ}$} & \multirow[t]{3}{*}{$\mathrm{RP}$} & \multirow[t]{3}{*}{ IC (95\%) } & \multirow[t]{3}{*}{$P$} \\
\hline & & \multicolumn{2}{|c|}{ Sim } & \multicolumn{2}{|c|}{ Não } & & & & \\
\hline & & $\mathrm{N}^{\circ}$ & $\%$ & $\mathrm{~N}^{\circ}$ & $\%$ & & & & \\
\hline \multirow[t]{2}{*}{ Abastecimento de água } & Não & 4 & 16,0 & 21 & 84,0 & 25 & 1,04 & 0,$42 ; 2,60$ & 0,935 \\
\hline & Sim & 93 & 15,4 & 511 & 84,6 & 604 & 1 & & \\
\hline \multirow[t]{2}{*}{ Tratamento da água no domicílio } & Não & 37 & 18,1 & 167 & 81,9 & 204 & 1,28 & 0,$88 ; 1,87$ & 0,191 \\
\hline & Sim & 60 & 14,1 & 365 & 85,9 & 425 & 1 & & \\
\hline \multirow[t]{2}{*}{ Coleta de esgoto } & Não & 65 & 16,5 & 328 & 83,5 & 393 & 1,21 & 0,$84 ; 1,76$ & 0,300 \\
\hline & Sim & 38 & 13,6 & 241 & 86,4 & 279 & 1 & & \\
\hline \multirow[t]{2}{*}{ Tipo de material da moradia } & Outros & 25 & 62,5 & 15 & 37,5 & 40 & 5,14 & 3,$72 ; 7,10$ & $<0,001$ \\
\hline & Tijolo & 72 & 12,2 & 520 & 87,8 & 592 & 1 & & \\
\hline \multirow[t]{2}{*}{$\mathrm{N}^{\circ}$ Pessoa/cômodo } & Acima 1,5 & 32 & 33,0 & 65 & 67,0 & 97 & 0,098 & $-0,15 ; 0,45$ & 0,656 \\
\hline & Até 1,5 & 189 & 35,3 & 346 & 64,7 & 535 & 1 & & \\
\hline \multirow[t]{2}{*}{ Idoso } & Presença & 5 & 22,73 & 17 & 77,27 & 22 & 1,09 & 0,$86 ; 1,36$ & 0,207 \\
\hline & Ausência & 118 & 16,12 & 614 & 83,88 & 732 & 1 & & \\
\hline
\end{tabular}

Notas: Ausência de informação: Abastecimento de água: 188; Tratamento de água no domicílio: 188; Coleta de esgotos: 145; Tipo de material: 185; Número de pessoas por cômodo: 185; Idoso: 63

Note: Without information: Water supply: 188; Water treated at home: 188; Presence of Sewer system: 145; type of building material: 185; \# of persons per room: 185; Elderly: 63 
Tabela 3 - Modelo final para ocorrência de doenças diarréicas, em crianças residentes no bairro Recreio de São Jorge, Guarulhos, SP, maio de 2005 a abril de 2006.

Table 3 - Final model for the occurrence of diarrhea diseases in children living in Recreio de São Jorge, Guarulhos, SP, May 2005 to April 2006.

\begin{tabular}{lccc}
\hline Variável & OR ajustada & IC (95\%) & $\mathrm{p}$ \\
\hline Desnutrição & 2,98 & $0,89-9,91$ & 0,076 \\
Idade da criança (4-9 meses) & 4,60 & $0,97-21,80$ & 0,054 \\
Idade da criança (10 meses e mais) & 7,53 & $1,71-33,13$ & 0,008 \\
Moradia * esgoto & 14,84 & $6,91-31,87$ & $<0,001$ \\
\hline
\end{tabular}

na construção da moradia que não o tijolo e inexistência de rede coletora de esgoto) e idade (10 meses e mais) como fatores de risco para ocorrência de diarreia (Tabela 3), sendo que as demais variáveis deixaram de ser significantes para o desfecho.

\section{Discussão}

O material utilizado na construção da moradia que não tijolo já havia sido identificado como fator de risco para ocorrência de diarreia na análise univariada, sugerindo que as condições precárias de moradia podem acarretar dificuldade de higienização no domicílio, fato que pode aumentar a ocorrência de doenças diarréicas. Por outro lado, esta variável é também um indicador de baixa renda familiar e de pobreza ${ }^{13}$.

A ausência de rede coletora de esgoto não havia se mostrado associada à ocorrência de diarreia na análise univariada; no entanto, identificou-se que há interação desta variável com o emprego de material precário na construção da moradia, elevando o risco de ocorrência de diarreia em quase 15 vezes para as crianças residentes em domicílios onde estas duas condições estavam presentes. Este resultado confirma o conceito de habitação saudável de Azeredo et al. ${ }^{14}$, que consideram que a habitação é um agente de saúde e se relaciona com o território geográfico e social, os materiais utilizados em sua construção, educação em saúde dos moradores e outras características do contexto do entorno.

A inexistência de acesso à rede coletora de esgotos interfere nas condições de saúde das crianças por poluir o ambiente e possibilitar a veiculação de doenças relacionadas a excretas, principalmente parasitoses, cuja maioria tem como sintoma principal a diarreia. A ausência de sistemas de esgotamento sanitário apropriado em assentamentos humanos, além de importante fonte de poluição de águas superficiais e subterrâneas, é fator de risco para a saúde da população, principalmente quando esta não tem conhecimento sobre a transmissão de doenças veiculadas pela água ou relacionadas com excretas. Giatti ${ }^{15}$, em pesquisa sobre saneamento em Iporanga (SP), verificou que os cursos de água pesquisados apresentaram índices microbiológicos que indicam a poluição por esgotos domésticos decorrente da insuficiência do saneamento básico local, considerando que $91 \%$ dos domicílios dispunham as excretas em fossas, em sua maioria rudimentares.

O cenário encontrado no Recreio de São Jorge reflete a situação de grande parte das áreas periurbanas da RMSP, que se caracteriza por alta taxa de crescimento populacional em áreas de mananciais ${ }^{16} \mathrm{ou}$ áreas de restrição legal, o que justifica o elevado número de ocupações irregulares, geralmente em condições precárias de moradia e falta de acesso ao saneamento básico. Tais condições acarretam degradação ambiental, principalmente em áreas de proteção de mananciais de abastecimento, além dos problemas de saúde verificados.

Outros estudos já haviam encontrado associação entre a residência em locais de invasão de terrenos ou de favela com a mortalidade neonatal em Campinas ${ }^{17} \mathrm{e}$ na região 
sul do $\mathrm{MSP}^{18}$, sugerindo que a localização de domicílios nestas áreas constitui-se em indicador de situação de exclusão social. Neste estudo, a interação identificada entre o material precário do domicilio e a ausência de sistema de esgoto sanitário, além de indicar situação de exclusão social, sugere que a presença conjunta destas condições pode aumentar a exposição aos patógenos causadores de doenças diarréicas, o que explica o elevado risco de ocorrência de diarreia entre crianças que apresentam estas condições de vida.

$\mathrm{O}$ acesso à água $\mathrm{e}$ a ocorrência de diarreia não apresentaram associação significativa com o desfecho, possivelmente porque quase toda a população tinha acesso à rede de abastecimento de água. Entretanto, segundo informações obtidas em campo pelos ACS, grande parte das famílias utilizava concomitantemente água proveniente da rede pública e de poços e córregos, pois a interrupção do abastecimento era muito frequente.

$\mathrm{O}$ acesso e o consumo de água proveniente de serviço público diminuem a probabilidade de ocorrência de doenças diarréicas, pois o padrão de potabilidade requerido no sistema garante a qualidade da água como fonte segura para consumo humano na entrada do domicílio. O mesmo não acontece quando o abastecimento resulta de fontes alternativas, de qualidade sanitária duvidosa e de volume nem sempre suficiente para atender às necessidades básicas $^{19}$. No entanto, problemas como intermitência do abastecimento criam condições satisfatórias para a infiltração de patógenos na rede de abastecimento de água, pois determinam pressões negativas no interior da rede ${ }^{20}$ e possibilitam o armazenamento da água em reservatórios domiciliares precários e sem higienização, que representam fatores de vulnerabilidade à qualidade da água do sistema público.

Checkley et al. ${ }^{21}$, em estudo realizado em área periurbana de Lima, Peru, concluíram que crianças jovens expostas a condições precárias de saneamento, considerando a fonte de água, a localização do reservatório e o destino dos esgotos, apresentaram $54 \%$ mais episódios de diarreia do que crianças não expostas. Estudo sumarizado por Briscoe $^{22}$ em áreas periurbanas da Ásia encontrou como resultado redução de $33 \%$ nas doenças diarréicas em crianças supridas por rede pública de abastecimento de água e de esgotamento sanitário.

Fatores de vulnerabilidade da rede pública, tais como frequente intermitência do serviço de abastecimento e existência de ligações clandestinas, os quais expõem a água à contaminação; ou fatores de vulnerabilidade domiciliar, como falta de limpeza dos reservatórios domiciliares, transporte e armazenamento em recipientes não adequados, introdução de objetos nos recipientes de armazenamento de água e hábitos e práticas não adequadas de higiene dos usuários, configuram-se como situações de risco para a contaminação da água.

Com relação às características das crianças, observou-se que a idade 10 meses e mais estava associada à ocorrência de diarreia; nesta idade já houve a introdução de outros alimentos na dieta infantil, que, sem os cuidados de higiene necessários em seu preparo, se apresentam como situação de risco à ocorrência de diarreia. Além disso, as crianças deste grupo passam a ter maior mobilidade, aumentando a chance de contaminação ambiental. Com relação ao grupo etário de 4 a 9 meses, observou-se um aumento de mais de 4 vezes do risco de diarreia, porém este não foi estatisticamente significante, sugerindo que a passagem do aleitamento exclusivo para misto pode ter algum efeito sobre a diarreia, confirmando a recomendação da $\mathrm{WHO}^{23}$ para o aleitamento materno exclusivo em crianças de até seis meses de idade, pois esta prática está fortemente relacionada com o aumento da sobrevivência infantil e a diminuição do risco de incidência de algumas doenças, particularmente as diarréicas.

Inicialmente, na análise multivariada, a desnutrição estava associada à ocorrência de diarreia; apenas na última etapa de modelagem esta variável foi excluída do modelo. Vários estudos mostram existir 
associação entre desnutrição e doenças diarréicas ${ }^{24}$. Observou-se colinearidade entre a presença de idosos na família e desnutrição, o que pode sugerir que estas crianças podem pertencer a unidades familiares não tradicionais e que tais famílias possam apresentar características socioeconômicas mais desfavoráveis ${ }^{25}$.

\section{O uso das informações do Programa Saúde da Família em estudos de saúde ambiental}

Os resultados obtidos mostram que as informações do SIAB podem se constituir em importante fonte de dados para identificar populações que vivem situações de risco ambiental. Frequentemente, estudos que envolvem saúde e ambiente têm esbarrado na dificuldade de obtenção de dados locais e específicos. Em muitos casos as informações disponíveis estão agregadas ao nível municipal, o que pode mascarar a presença de condições de vida heterogêneas e, como consequência, dificultar a apreensão de condições de risco existentes no nível local, realidade frequente nos aglomerados urbanos do país. Nesse sentido, o SIAB pode representar importante fonte de dados para estudos que privilegiam a inter-relação ambiente-saúde, uma vez que disponibiliza dados sistemáticos sobre saúde da família, condições da moradia e acesso a serviços básicos de saneamento.

No entanto, o emprego dos dados do SIAB em estudos de saúde ambiental apresenta algumas limitações, que poderiam facilmente ser aprimoradas. Há necessidade de melhorar o registro de dados, principalmente das informações ambientais que apresentaram valores abaixo de $10 \%$, sendo que estas informações podem ser facilmente obtidas. As informações deveriam ter atualização periódica. A coleta dos dados do cadastro das famílias em geral ocorre na primeira visita dos ACS; entretanto, as condições ambientais das moradias são dinâmicas e podem se alterar com o passar do tempo. Contudo, esta dificuldade pode ser contornada pelo pesquisador, que poderia vir a utilizar uma amostra de famílias para inspeção domiciliar para atualizar as informações de cadastro ou mesmo adicionar informações às existentes na ficha de coleta de dados para a obtenção de indicadores de acordo com as necessidades do estudo.

Tendo em vista que as informações do SIAB podem ser utilizadas em estudos de ambiente-saúde, com a incorporação de variáveis que melhor caracterizem as condições ambientais dos domicílios e que podem ser facilmente obtidas. Como sugestões de aprimoramento poderiam ser incluídos dados sobre procedência da água de outras fontes, que não a rede pública, como poços ou nascentes; aspectos relativos à qualidade da água acessada; frequência da intermitência do abastecimento de água; existência, tipo e quantidade de animais domésticos; condições do entorno da moradia, principalmente do quintal; se a família reside em área de invasão ou de vulnerabilidade ambiental; práticas de reaproveitamento do lixo; e emprego de práticas de higiene domiciliar. Na ficha de acompanhamento da criança, são importantes a idade e a ocupação da mãe ou responsável.

Em estudo sobre indicadores de saúde ambiental, Calijuri et al. ${ }^{26}$ propuseram como variáveis de saneamento a falta de água no domicílio, para verificar a frequência de intermitência do serviço, e o tipo de abastecimento dos domicílios não ligados à rede. Além disso, variáveis que permitem identificação de informações sobre a presença de animais, moscas e plantas no domicílio para o grupo saúde ambiental, são interessantes para serem utilizadas no cadastro dos domicílios do PSF.

\section{Conclusões}

Em grandes aglomerados urbanos, os indicadores de saneamento básico podem não ser eficientes para identificar populações que vivem situações de risco para a ocorrência de diarreia. As informações coletadas pelo Programa de Saúde da Família podem se constituir em excelente ferramenta para identificação de núcleos 
populacionais, onde ainda existem parcelas da população vivendo em precárias condições de habitação e sem acesso a sistemas de saneamento básico. Nessas condições, o estudo verificou que o risco de ocorrência de diarreia em crianças é quase 15 vezes maior do que entre aquelas que vivem em condições adequadas de habitação e saneamento.

\section{Aspectos éticos}

O Comitê de Ética em Pesquisa de Saúde Pública da Universidade de São Paulo COEP analisou e aprovou o Protocolo de Pesquisa $\mathrm{N}^{\circ} 1435$, de acordo com os requisitos da Resolução CNS/196/96 e suas complementares.

\section{Referências}

1. Özkan S, Tüzün H, Görer N, Ceyhan M, Aycan S, Albayrak S. Water usage habits and the incidence of diarrhea in rural Ankara, Turkey. Trans Soc Trop Med Hyg 2007; 101(11): 1131-5.

2. UNICEF-United Nations International Children's Emergency Fund; WHO-World Health Organization. Diarrhea: why children are still dying and what can be done. UNICEF; WHO: Geneva; New York; 2009.

3. Fewtrell L, Kaufmann RB, Kay D, Enanoria W, Haller L, Collford JM Jr. Water, sanitation and hygiene interventions to reduce diarrhea in less developed countries: a systematic review and meta-analysis. Lancet Infec Dis 2005; 5(1): 42-52.

4. Bozkurt AI, Ozgür S, Ozçirpici B. Association between household conditions and diarrheal diseases among children in Turkey: a cohort study. Pediatr Int 2003; 45(4): 443-51.

5. Moe CL, Rheingans RD. Global challenges in water, sanitation and health. JWater Health 2006; 4: 41-57.

6. Teixeira JC, Pungirum MEM de C. Análise da associação entre saneamento e saúde nos países em desenvolvimento da América Latina e do Caribe, empregando dados secundários do banco de dados da Organização Pan-Americana de Saúde - OPAS. Rev Bras Epidemiol 2005; 8(4): 356-76.

7. Trevett AF, Carter RC, Tyrrel SF. The importance of domestic water quality management in the context of fecal-oral disease transmission. JWater Health 2005; 3(3): 259-70.

8. Brasil. Constituição da República Federativa do Brasil (1988). Brasília: Senado; 1988.

9. Blakely T, Hales S, Kieft C, Wilson N, Woodward A. The global distribution of risk factors by poverty level. Bull World Health Organ 2005; 83(2): 118-26,.

10. Andreazzi MAR, Barcellos C, Hacon S. Velhos indicadores para novos problemas: a relação entre saneamento e saúde. Rev Panam Salud Publica 2007; 22(3): 211-7.

11. Heller L. Saneamento e saúde. Brasília: OPAS; 1997.
12. Kelsey JL, Whittemore, AS, Evans, AS, Thompson, WD. Methods in observational epidemiology. New York: Oxford University Press; 1996.

13. IBGE-Instituto Brasileiro de Geografia e Estatística. Censo 2000. Rio de Janeiro: IBGE; 2001.

14. Azeredo CM, Cotta RMM, Schott M, Maia T de M, Marques ES. Avaliação das condições de habitação e saneamento: a importância da visita domiciliar no contexto do Programa Saúde da Família. Ciênc Saúde Coletiva 2007; 12(3): 743-53.

15. Giatti LL, Rocha, AA, Santos FA dos, Bitencourt SC, Pieroni SR de M. Condições de saneamento básico em Iporanga, Estado de São Paulo. Rev Saúde Pública 2004; 38(4): 571-7.

16. Porto M. Recursos hídricos e saneamento na Região Metropolitana de São Paulo: um desafio do tamanho da cidade. Brasília: Banco Mundial; 2003.

17. Almeida, SDM, Barros, MBA. Atenção à saúde e mortalidade neonatal. Rev Bras Epidemiol 2004; 7(1): 22-35.

18. Shoeps D, Almeida MF de, Alencar GP, França Jr I, Novaes HMD, Siqueira AAF et al. Fatores de risco para a mortalidade neonatal precoce. Rev Saúde Pública 2007; 41(6): 1013-22.

19. Razzolini MTP, Günther WMR. Impacto na saúde das deficiências de acesso à água. Saúde Soc 2008; 17(1): 2132 .

20. Lee EJ, Schwab KJ. Deficiencies in drinking water distribution systems in developing countries. JWater Health 2005; 3(2): 109-27.

21. Checkley W, Gilman R, Black RE, Epstein LD, Sterling CR, Moulton LH. Effect of water and sanitation on childhood health in a poor Peruvian peri-urban community. Lancet 2004; 363 (9403): 112-8.

22. Briscoe J. Abastecimento de agua y servicios de saneamiento; su función em la revolución de la supervivencia infantil. Bol Oficina Sanit Panam 1987; 103(4): 325-39. 
23. WHO-World Health Organization. Infant and young child feeding: a tool for assessing national practices, policies and programmers. 2003. Disponível em http:// www.who.int/ nutrition/publications/infantfeeding/ [Acessado em 30 de janeiro de 2007]

24. WHO-World Health Organization; UNICEF-United Nations International Children's Emergency Fund; USAID-United States of America Agency International Development; SIDA-Sweden International Development Agency. Innocent declaration on the protection, promotion and support of breastfeeding. Florence: WHO/ UNICEF; 1990.
25. Camarano, AA. O idoso brasileiro no mercado de trabalho. Instituto Brasileiro de Pesquisa Econômica Aplicada - IPEA, Rio de Janeiro: 2001. Disponível http://www.ipea.gov.br/pub/td/td_2001/td_0830.pdf [Acessado em 18 de abril de 2008]

26. Calijuri ML, Santiago A da F, Camargo R de A, Moreira Neto RF. Estudo de indicadores de saúde ambiental e de saneamento em cidade do Norte do Brasil. Eng Sanit Amb 2009; 14(1): 19-28.

Recebido em: 26/08/10

Versão final apresentada em: 16/03/11

Aprovado em: 22/03/11 